\title{
Beyond paleoclimate ping pong
}

Nils Weitzel', C. Brierley², J. Bühler', M. Chevalier ${ }^{3}$, B. Ellerhoff', V. Skiba ${ }^{4}$ and K. Rehfeld ${ }^{1,5}$

Heidelberg, Germany, and online, 5-7 July 2021

A key question of the paleoclimate community is how paleoclimate data can be used to evaluate long-term predictability in climate models. How can we improve estimates of past climate variability and our understanding of the state and timescale dependency of Earth's climate? "Ping pong" serves as a metaphor to describe the back-and-forth in comparing paleoclimate data with model simulations. This is a core challenge in climate research, which requires a better understanding of proxies as well as the consequences of neglected or poorly simulated processes in climate models.

To address this question, this Climate Variability Across Scales (CVAS;

pastglobalchanges.org/cvas) workshop (pastglobalchanges.org/calendar/26970) brought together a diverse pool of $\sim 60$ scientists, ranging from early-career scientists to experienced experts from various fields and different working groups, including CVAS, Speleothem Isotopes Synthesis and AnaLysis (SISAL; pastglobalchanges.org/ sisal), 2k Network (pastglobalchanges.org/2k), and the PAGES-endorsed Paleoclimate Modelling Intercomparison Project (PMIP; pmip.Isce.ipsl.fr). One half participated online and the other half gathered at the "Internationales Wissenschaftsforum" in Heidelberg, Germany. Keynote talks focused on climate variability on different temporal and spatial scales, best practices for the joint use of models and proxies, the role of paleoclimate in future predictions, and the state of the art in the analysis and interpretation of various proxy types. Discussions resulting from these presentations continued in three working groups.

The first group discussed philosophical questions regarding the design and impact of data-model comparison studies, summarized in Figure 1. Among these were what insights into climate can be gained from datamodel comparison, and how both sources of information can be leveraged. The importance of formulating a clear hypothesis prior to the comparison was stressed, as well as the need to explain experimental choices and assumptions such that the scope and limitations of the respective analysis are clearly defined. Consequently, techniques for a rigorous treatment of uncertainties are required and due to various uncertainties on both sides, data and models are not necessarily expected to "agree".

The second group formulated a research project based on the recommendations from the first group. Inspired by the keynote talks, the group decided to study the temperature-hydroclimate relationship in the tropics and test the hypothesis of positive covariability between the two. The participants identified suitable databases (e.g. PAGES2k Consortium 2017; Konecky et al. 2019; Comas-Bru et al. 2020) for a multi-archive and multi-proxy approach. Comparison against isotope-enabled simulations (e.g. Bühler et al. 2021) is planned. Key questions that the group identified include whether emerging isotope-enabled simulations

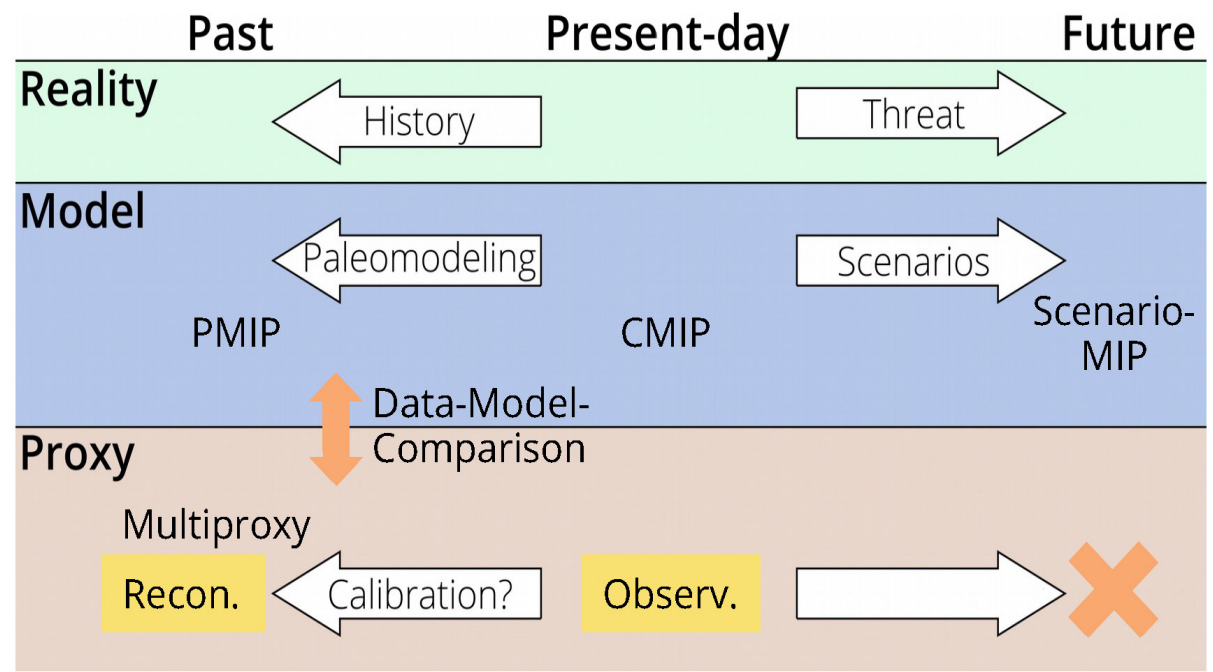

Figure 1: Key components and challenges of data-model comparison. The relevant tools, variables, intercomparison projects, and challenges (in orange) are illustrated with respect to the targeted time ranges. The workshop specifically addressed the overarching question of how paleoclimatology can contribute to solving research questions on future climate scenarios. facilitate more robust data-model comparisons, and how multiple archives and simulations can be used to understand the underlying mechanisms controlling the covariability of hydroclimate and temperature.

The third group started with the conundrum of reported agreement of global mean temperature variability in models and proxies on decadal-to-centennial scales (e.g. Neukom et al. 2019), whereas reconstructed local surface temperature variability is higher than in simulations (e.g. Laepple and Huybers 2014). The group reviewed the literature, with a focus on the spatial and temporal scales of interest. Finally, the group collected and assessed potential reasons to explain the conundrum, including effects from an overestimation of spatially uncorrelated variability in temperature reconstructions, misspecification of the spatial correlation structures in models, and the suppression of variability by climate field reconstruction methods. The group plans to expand the literature review and develop research protocols to quantify the contributions of identified potential explanations.

For most participants, the workshop was the first experience with a hybrid conference format, and the feedback was quite positive. We emphasize the importance of an appropriate technical infrastructure on site and the prior set-up of a clear workshop structure. The use of a virtual communication platform and shared working documents helped to connect virtual and on-site participants.

\section{AFFILIATIONS}

IInstitute of Environmental Physics, Heidelberg University, Germany

2Department of Geography, University College London, UK

${ }^{3}$ Institute for Geosciences, University of Bonn Germany

${ }^{4}$ Potsdam Institute for Climate Impact Research, Germany

${ }^{5}$ Geo- und Umweltforschungszentrum, Tübingen University

CONTACT

NilsWeitzel: nweitzel@iup.uni-heidelberg.de

REFERENCES

Bühler J et al. (2021) Clim Past 17: 985-1004

Comas-Bru L et al. (2020) Earth Syst Sci Data 12: 2579-2606

Konecky BL et al. (2019) Earth Syst Sci Data 12: 2261-2288 Laepple T, Huybers P (2014) Proc Natl Acad Sci USA 111 16682-16687

Neukom R et al. (2019) Nat Geosci 12: 643-649

PAGES2k Consortium (2017) Sci Data 4: 170088 